\title{
Alterations in gait velocity and grip strength of stroke survivors following a 12-week structured therapeutic exercise programme
}

\author{
Hammed I. Adebisi ${ }^{1}$, Adodo S. Monikhe ${ }^{2}$, Agwubike E. Okey ${ }^{2}$ \\ ${ }^{1}$ Department of Physiotherapy, University of Benin Teaching Hospital, Benin City, Nigeria; ${ }^{2}$ Department of Human \\ Kinetics and Sports Science, University of Benin, Benin City, Nigeria
}

\section{Summary}

Study aim: This study investigated the alterations in gait velocity and grip strength of stroke survivors following a structured therapeutic exercise programme (STEP).

Material and methods: This was a pre-test, post-test experimental study of the effectiveness of a 12-week STEP on gait velocity and grip strength of stroke survivors. A total of 30 hemiparetic stroke survivors participated in the study. The instrument for this study was an adaptation of a training protocol for the training and assessment of gait velocity and grip strength. The participants underwent a 12-week STEP of a frequency of 3 times per week and the training programme focused on exercises aimed at improving the gait velocity and grip strength of the participants. The gait velocity and grip strength were measured before and after the training. Data generated were analysed using descriptive statistics of mean and standard deviation to summarize the profile of the participants. The analysis of variance for repeated measures (ANOVA) was used to test the hypotheses. Statistical significance was accepted for a $\mathrm{p}$ value of $<0.05$.

Results: The outcome of this study showed that the STEP had significant $(\mathrm{p}<0.05)$ effects on the gait velocity and grip strength of stroke survivors. Meanwhile, there was no significant $(\mathrm{p}>0.05)$ effect of haemorrhagic and ischaemic groups of stroke survivors on gait velocity and grip strength.

Conclusion: It was therefore concluded that the use of the STEP can substantially improve the gait velocity and grip strength of stroke survivors.

Keywords: Exercise therapy - Gait velocity - Grip strength - Stroke survivors

\section{Introduction}

Stroke, as defined by Sims, et al. [14], is a rapidly developing loss of brain function(s) due to disturbance in the blood supply to the brain. According to the authors, it could be due to ischaemia, which is a lack of blood flow, caused by blockage (thrombosis or arterial embolism) or haemorrhage, which is leakage of blood into the brain. The physical manifestation is paresis or paralysis (partial or complete weakness) of the muscles of the limbs of the contralateral side, otherwise known as the hemiparetic or hemiplegic side. It is important to note that patients afflicted with stroke are faced with a lot of challenges. According to Gordon [2], such challenges include reduced functional capacity, motor activity intolerance, muscle atrophy, partial paralysis, residual gait deviation, anxiety, job/economic stress and, for some, an overwhelming sense of uncertainty. These are dependent on several variables such as age, gender, severity of neurological involvement and the like.

Moreover, walking ability is one of the most important functions because independent ambulation is essential for community reintegration and social participation. Thus, gait training accounts for a large proportion of time spent in stroke rehabilitation. So, restoration of normal movements of the trunk, pelvis, and lower extremity while walking, improving symmetry and weight bearing on the paretic side, and to establish an energy-efficient walk, are the most important goals of gait training in stroke patients [23]. Gait speed among stroke survivors has enjoyed a lot of research over the years with stimulating results. Several authors have reported varying results ranging from significant findings to non-significant findings. However, the baseline is that the gait rehabilitation exercise programme has been reported to have brought about improvement in 
the gait speed of stroke survivors [7, 20, 27]. In fact, dependence in mobility is one of the primary reasons for admission for inpatient rehabilitation after stroke [4]. In addition, many factors can influence the level of limitation in walking activity, and these include learning ability, coping skills, motivation, medical co-morbidities, physical endurance levels, family support, housing and the amount and type of rehabilitation training [13]. Consequently, hemiparetic gait is characterized by slow and asymmetric steps with poor selective motor control delayed and disrupted equilibrium reactions and reduced weight bearing on the paretic limb [13, 21]. Likewise, smooth and symmetric forward progression of the body is impaired, with a large variation in gait patterns related to the degree of recovery. Well-controlled intra-limb and inter-limb coordination is replaced by mass limb movement patterns (synergies) on the paretic side requiring compensatory adjustments of the pelvis and non-paretic side. Compensatory movements necessary for ambulation produce abnormal displacement of the centre of gravity, resulting in increased energy expenditure [6].

On the other hand, reliable and valid evaluation of hand strength can provide an objective index of general upper body strength. Grip strength is the result of forceful flexion of the finger joints with the maximal voluntary force that a person is able to exert under normal biokinetic conditions. Therefore, grip strength is an important component in performing precise and refined fine motor activities. It is one of the commonest measurements used as a part of physical assessment of various diseases affecting musculoskeletal, neuromuscular and cardiorespiratory systems in children and the elderly population. It also provides selective information to estimate the nutritional status and overall muscle strength of an individual [10]. However, a decrease in grip strength can cause significant functional limitations which could lead to decreased basic and instrumental activities of daily living, thereby diminishing the quality of life of an individual. This is because hand functionality is considered to be vital in most of the activities involving the upper limb, be it writing, eating, carrying loads, lifting objects, or opening and closing doors, to name a few. Grip strength is often used in medicine as a specific test for upper limb strength. The purpose of this testing is diverse, including to diagnose diseases, to evaluate and compare treatments, to document progression of muscle strength, and to provide feedback during the rehabilitation process as a measure of indicating the level of hand function and fatigue.

Moreover, strength training is one intervention with the potential to improve function among stroke survivors. Various studies have demonstrated improvement of varying degrees of the muscular strength of stroke survivors. Harris, et al [3] reported a significant effect of strength training on upper-limb function in studies including subjects with moderate and mild upper-limb impairment among stroke survivors. It is particularly important to remember that exercise therapy has the capacity to increase strength and develop muscle [8]. Substantial evidence has pointed to the obvious effects of therapeutic exercise programmes on gait velocity and grip strength of stroke survivors $[5,11$, $16,17,19]$. However, it is worth noting that gradients or clinical characteristics of stroke morbidity and other clinical factors which are said to affect the outcome of exercise therapy are never the same across communities in the same country, and nor are they the same across countries. For this reason, the effects of exercise therapy on gait velocity and grip strength of stroke survivors in this locality may not be the same as found elsewhere. This study was therefore conducted to establish the efficacy of a structured therapeutic exercise programme (STEP) on gait velocity and grip strength among Nigerian stroke survivors.

\section{Research hypotheses}

The following hypotheses were formulated and tested at the 0.05 significance level.

1. There is no significant difference in the gait velocity of stroke survivors prior to and following a 12-week STEP.

2. There is no significant difference in the grip strength of stroke survivors prior to and following a 12-week STEP.

3. There is no significant difference between haemorrhagic and ischaemic stroke survivors in their gait velocity prior to and following a 12-week STEP.

4. There is no significant difference between haemorrhagic and ischaemic stroke survivors in their grip strength prior to and following a 12-week STEP.

\section{Material and Methods}

\section{Research design}

This study was a pre-test, post-test experimental design concerning the alterations in gait velocity and grip strength of stroke survivors following a 12-week STEP.

\section{Population}

The population for this study included 65 hemiparetic stroke survivors between the biological ages of 45 and 75 , who were receiving treatment at the Physiotherapy Department of the University of Benin Teaching Hospital from $1^{\text {st }}$ June to $31^{\text {st }}$ December, 2015.

\section{Sample size and sampling technique}

The sample for this study comprised 30 hemiparetic stroke survivors receiving treatment at the Physiotherapy Department of the University of Benin Teaching Hospital. The purposive sampling technique was used to select 
the 30 hemiparetic stroke survivors comprising 15 hemorrhagic and 15 ischaemic stroke patients based on the following criteria:

Inclusion criteria:

- The participant must be ambulant without a walking aid.

- The participant's grip strength must be good enough to compress the handgrip exerciser.

Exclusion criteria:

- Participants with communication deficits and cognitive impairment.

- Participants with an upper-limb fracture in the past 6 months.

\section{Data collection instruments}

The instrument for this study was a STEP for training and assessment of gait and muscular strength in the Physiotherapy Department of the University of Benin Teaching Hospital.

\section{Validity and reliability of data collection instrument}

The test instrument was validated for the training and assessment of upper and lower limb strength by experts in physiotherapy and exercise physiology, and its reliability was calculated to be 0.81 to $0.88(\mathrm{r}=0.81-0.88)$ using the split-half method.

\section{Data collection procedure}

The study received ethical approval to conduct this study from the Research Ethics Committee of the University of Benin Teaching Hospital, Nigeria. Prior to the STEP, a detailed explanation of the test, training programme and; the objectives and intricacies of the study was provided to the participants and then the participants signed a participant's informed consent form before participating in this study. Thereafter, the participants were made to undergo a 12-week STEP of a frequency of 3 times per week (Monday, Wednesday, and Friday) with each session lasting for 50 minutes at $70 \%$ one-repetition maximum $(70 \% 1 \mathrm{RM})$. The training programme focused on exercises aimed at improving the gait velocity and grip strength of the participants, in order to increase their functional status to meet their daily challenges. The gait velocity and grip strength of the participants were measured prior to and after the training.

\section{Gait velocity training}

Aim: To increase the mobility of the participants.

Equipments: An open space within the gymnasium/department and a stopwatch.

Procedure: Each participant was made to walk a distance of $10 \mathrm{~m}$ from one end of the gymnasium to the other.

Instruction: From the starting point, each participant was instructed to walk without a walking aid as fast as he/ she could without running, to the end point. The process was repeated three times with three minutes rest between the sets. Time taken to cover each $10 \mathrm{~m}$ distance was recorded in seconds.

Scoring: The total distance covered $(30 \mathrm{~m})$ by each participant was divided by the total time taken. The result was, however, recorded as the gait velocity.

\section{Grip strength training}

Aim: To improve the grip strength of the affected hand.

Equipment: Hand grip exerciser.

Procedure: Participants were made to compress the hand grip exerciser 50 times as hard as they could with the affected hand.

Instruction: Each participant was instructed to compress or grip the test equipment 50 times as hard as he/she could continuously with the affected hand. The process was repeated three times with three minutes rest between the sets.

Scoring: Grip strength was measured using a Camry Electronic Hand Dynamometer (Model: EH101). This device comes with dual scale readout of forces in kilograms and pounds. However, all readings were recorded in kilograms in the present study. To standardize the test, the following guidelines were established: the arm positioning followed the American Society of Hand Therapists guidelines [1], with the participant comfortably seated and the shoulder slightly forward with the elbow flexed at a $90^{\circ}$ angle. The forearm and wrist were in a neutral position. Three maximum efforts were performed alternately for the affected hand, with three-second contractions and ten-second rest periods between the attempts. The best of three attempts was recorded. The following test instructions were provided: "you must squeeze the handle as hard as possible keeping both your body and arm in position". The same tone was used during the briefings, and no verbal encouragement was offered. The instrument calibration was assessed periodically throughout the study.

\section{Method of data analysis}

Data generated were analyzed using descriptive statistics of mean and standard deviation to summarize the profile of the participants. Then, the analysis of variance for repeated measures (ANOVA) was used to test the hypotheses. Statistical significance was accepted for $\alpha=0.05$. All the analyses were performed using the IBM SPSS Statistics, version 20.0.

\section{Results}

The results of the grip strength and gait speed registered before and after the 12-week STEP programme are shown in Table 1. 
Table 1. Mean $( \pm \mathrm{SD})$ pre- $(0)$ and post- (1) programme values of gait velocity $(\mathrm{V})$ and handgrip force (HG) for stroke survivors

\begin{tabular}{lcccc}
\hline Groups & $\mathrm{V} 0[\mathrm{~m} / \mathrm{s}]$ & $\mathrm{V} 1[\mathrm{~m} / \mathrm{s}]$ & $\mathrm{HG} 0[\mathrm{~N}]$ & $\mathrm{HG} 1[\mathrm{~N}]$ \\
\hline Ischaemic $(\mathrm{n}=15)$ & $0.532 \pm 0.293$ & $0.945 \pm 0.302$ & $101 \pm 86$ & $205 \pm 217$ \\
Haemorrhagic $(\mathrm{n}=15)$ & $0.544 \pm 0.296$ & $0.949 \pm 0.351$ & $89 \pm 80$ & $181 \pm 188$ \\
\hline All & $0.538 \pm 0.289$ & $0.947 \pm 0.322$ & $95 \pm 82$ & $193 \pm 200$ \\
\hline
\end{tabular}

$\mathrm{n}=$ frequency within a group or subgroup.

\section{Hypothesis testing}

Hypothesis 1: Rejected: there was a significant increase in gait velocity $\left(\mathrm{F}_{1,28}=108.6, \mathrm{p}<0.001 ; \eta^{2}=0.795\right)$.

Hypothesis 2: Rejected: there was a significant increase in grip strength $\left(\mathrm{F}_{1,28}=17.72, \mathrm{p}<0.001 ; \eta^{2}=0.388\right)$.

Hypothesis 3: Not rejected: there was no effect of group on gait velocity $\left(\mathrm{F}_{1,28}=0.006, \mathrm{p}=0.9883 ; \eta^{2}=0.00002\right)$.

Hypothesis 4: Not rejected: there was no effect of group on grip strength $\left(\mathrm{F}_{1,28}=0.126, \mathrm{p}=0.7253 ; \eta^{2}=0.0045\right)$.

\section{Discussion}

The STEP was found in the present study to have a substantive effect on the gait velocity of stroke survivors. This finding contrasts with the study of Pomeroy et al. [12], who reported no significant difference in the gait velocity between the treatment and the control groups among stroke survivors following the STEP. In a related study carried out by States et al. [15], on overground physical therapy gait training for chronic stroke patients with mobility deficits, an insignificant finding was also reported. Furthermore, the finding of this study is also not in agreement with the study of Moseley et al. [9], who reported insignificant effects of treadmill training and body support for walking after stroke. These contrasting findings might be related to variation in study methodology including subject characteristics or differences in the training protocols and measurements of gait velocity. This could also be as a result of differences in the gradients/chronicity or clinical characteristics of stroke morbidity. However, there are several other studies which demonstrated significant effects of therapeutic exercise training on gait velocity of stroke survivors and thus agree with the findings of the present study. For example, Peurala et al. [11] examined the effectiveness of body-supported gait training and floor walking in 45 ambulatory patients with chronic stroke, and observed faster gait velocity after the intensive rehabilitation programme. Likewise, gait velocity of stroke survivors with hemiparesis during the six-minute walk has also been reported to have increased by $62 \%$ following a 12 -week therapeutic intervention [5].

Similarly, the findings of this study on the effectiveness of STEP on grip strength of stroke survivors did not enjoy the support of many researchers who found and reported no significant effect of the programme. For example, Suputtitada et al. [19] found no significant difference in grip strength between groups measured by a dynamometer after 2 weeks of bimanual upper extremity training among chronic stroke patients. Moreover, Stinear et al. [17] studied 9 stroke patients, who received active-passive bimanual movement therapy using a purpose-built machine that facilitated passive rhythmical flexion/extension of the paretic wrist using active flexion/extension of the less-affected wrist. They also reported insignificant improvement in grip strength at posttreatment. The same result was reported by Stoykov et al. [18] after 8 weeks of arm training among 24 stroke survivors with chronic stroke and moderate upper limb impairment who received functional bilateral or unilateral arm training. That is, there were no significant differences in grip strength between groups after treatment 3 times per day for 8 weeks. Furthermore, other researchers [16, 22] also reported insignificant an improvement in the paretic grip strength of stroke survivors measured by a hand dynamometer after a few weeks of arm training. The discrepant findings among studies might also be due to methodological nuances, such as the timing of measurements, statistical modelling, etc. However, the STEP had a different effect between haemorrhagic and ischaemic stroke survivors in their gait velocity in the present study. The significant difference in the gait velocity between haemorrhagic and ischaemic stroke survivors could be a result of differences in the pathophysiology of the two types of stroke (haemorrhagic and ischaemic), which could cause variation in motor weakness, thereby resulting in a difference in walking velocity.

However, the limitations of this study were that only the STEP was considered because of difficulty in monitoring other activities of the participants, such as prescribed medications and other home programmes, and the severity of stroke was not taken into consideration due to inadequate clinical investigations and incomplete documentation.

\section{Conclusions}

It is therefore concluded that:

- The participants' gait velocity and grip strength improve substantially following STEP. 
- The STEP equally affects the gait velocity and grip strength of both haemorrhagic and ischaemic groups of stroke survivors.

Based on the findings, it is recommended that:

1. The STEP should be considered a key element in the management of stroke survivors, thereby enhancing clinical outcome.

2. It is necessary to have a control group in further studies.

Conflict of interest: Authors state no conflict of interest.

\section{References}

1. Fess E.E. (1992) Grip Strength. American Society of Hand Therapists. 2nd edition, Chicago.

2. Gordon N.F. (2004) American Heart Association: Physical activity and exercise recommendations for stroke survivors. Circulation, 109: 2031-2041.

3. Harris J.E., J. Janice (2010) Strength training improves upper-limb function in individuals with stroke. Stroke AHA, 41: 136-140.

4. Hendricks H.T., J. Van Limbeek, A.C. Geurts, M.J. Zwarts (2002) Motor recovery after stroke: a systematic review of the literature. Arch. Phys. Med. Rehabil., 83: 1629-1637.

5. Jorgensen J.R., D.T. Bech-Pedersen, P. Zeeman, J. Sorensen, L.L. Anderson, M. Schonberger (2010) Effect of intensive outpatient physical Training on gait performance and Cardiovascular Health in people with Hemiparesis after stroke. Physi. Ther., 90(4): 527-537.

6. Kim C.M., J.J. Eng (2004) Magnitude and pattern of 3D kinematic and kinetic gait profiles in persons with stroke: relationship to walking speed. Gait Posture, 20: 140-146.

7. Kramers de Quervain I.A., S.R. Simon, S. Leurgans, W.S. Pease, D. McAllister (1996) Gait Patterns in the Early Recovery Period after Stroke. J. Bone Joint Surg., 78: 1506-1514.

8. Kryger A.I., J.L. Andersen (2007) Resistance training in the oldest old: Consequences for muscle strength, fiber types, fiber size, and MHC isoforms. Scand J. Med. Sci. Sports, 17: 422-430.

9. Moseley A.M., A. Stark, I.D. Cameron, A. Pollock (2005) Treadmill training and body weight support for walking after stroke. Cochrane Database Syst Rev; Oct 19, (4), CD 002840.

10. Norman K.N., M.C. Stobaus, J.D. Gonzalez, M. Schulzke, M. Pirlich (2011) Hand grip strength: outcome predictor and marker of nutritional status. Clin. Nutr., 30: 135-142.

11. Peurala S.H., I.M. Tarkka, K. Pitkanen, J. Sivenius (2005) The effectiveness of body weight-supported gait training and floor waking in patients with chronic stroke. Arch. Phys. Med. Rehabil., 86(8): 1557-1564.
12. Pomeroy V.M., M. Evans, M. Falconer, D. Jones, E. Hill, G. Giakas (2001) An exploration of the effects of weighted garments on balance and gait of stroke patients with residual disability. Clin. Rehabil., 15(4): 390-397.

13. Roth E.J., R.L. Harvey (2000) Rehabilitation of stroke syndromes. In: Braddom R.L. (ed.) Physical Medicine and Rehabilitation. 2nd ed. Philadelphia, Pa: WB Saunders; 1117-1163.

14. Sims N.R., H. Muyderman (2009) Mitochondria, oxidative metabolism and cell death in stroke. Biochim. Biophys. Acta, 1802(1): 80-91.

15. States R.A., Y. Salem, E. Pappas (2009) Overground gait training for individuals with chronic stroke: a cochrane systematic review. J. Neurol. Physther., 33(4): 179-186.

16. Stinear C.M., P.A. Barber, J.P. Coxon, M.K. Fleming, W.D. Byblow (2008) Priming the motor system enhances the effects of upper limb therapy in chronic stroke. Brain, 131: 1381-1390.

17. Stinear J.W., W.D. Byblow (2004) Rhythmic bilateral movement training modulates corticomotor excitability and enhances upper limb motricitypoststroke: a pilot study. J. Clin. Neurophysiol., 21(2): 124-131.

18. Stoykov M.E., G.N. Lewis, D.M. Corcos (2009) Comparison of bilateral and unilateral training for upper extremity hemiparesis in stroke. Neurorehabilitation and Neural Repair, 23(9): 945-53.

19. Suputtitada A., N.C. Suwanwela, S. Tumvitee (2004) Effectiveness of constraint-induced movement therapy in chronic stroke patients. J. Med. Assoc. Thai., 87(12): 1482-1490.

20. Tur B.S., Y.K. Gursel, G. Yavuzer, A. Kucukdeveci, T. Arasil (2003) Rehabilitation outcome of Turkish stroke patients: in a team approach setting. Int. J. Rehabil. Res., 26: 271-277.

21. Tyson S.F. (1999) Trunk kinematics in hemiparetic gait and the effect of walking aids. Clin. Rehabil., 13: 295-300.

22. Whitall J., S. McCombe Waller, K.H.C. Silver, R.F. Macko (2000) Repetitive bilateral arm training with rhythmic auditory cueing improves motor function in chronic hemiparetic stroke. Stroke AHA, 31: 2390-2395.

23. Yavuzer G., H. Gok, S. Ergin (2001) Spatiotemporal and kinematic gait characteristics of stroke patients. J. Rheum. Med. Rehab., 12: 148-152.

24. Yavuzer G., A. Kucukdeveci, T. Arasil, A. Elhan (2001) Rehabilitation of stroke patients: Clinical profile and functional outcome. Am. J. Phys. Med. Rehab., 80: 250-255.

\section{Received 10.06.2017 \\ Accepted 10.05.2018}

(C) University of Physical Education, Warsaw, Poland 